\title{
Origen dẹ la Dirección General de Docencia de Pregrado de la UAA
}

Departamento de Formación y Actualización Académica

\section{Resumen}

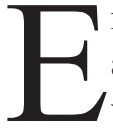
n el marco del 45 aniversario de la Universidad Autónoma de Aguascalientes, se presenta en este artículo los orígenes de la Dirección General de Docencia de Pregrado, como el área administrativa encargada de "diseñar y ejecutar las políticas y programas institucionales de apoyo a la docencia de pregrado y coordinar programas de apoyo a maestros y alumnos” (UAA, 2015: 29).

Palabras clave: docencia, pregrado, profesor, universidad.

\section{Introducción}

La docencia, investigación y difusión son las tres funciones sustantivas de una institución de educación superior. En el caso de la docencia, uno de los fines de la Universidad Autónoma de Aguascalientes es "impartir la enseñanza media y superior en el Estado de Aguascalientes" (UAA, 2015: 7), por lo cual el Estatuto de la Ley Orgánica plantea que "los fines de la universidad se centran en formar a los estudiantes desde una perspectiva humanista que enfatiza el desarrollo equilibrado e integral de las dimensiones de su persona, lo cual les permita desempeñarse exitosamente como futuros profesionistas y vivir la vida con plenitud y calidad" (UAA, 2015: 22).

Para lograr estos fines, el desarrollo de una docencia de calidad es imprescindible, que, de acuerdo con el Plan de Desarrollo Institucional 2016-2014, "tiene como propósito formar integralmente y con un alto sentido humanista a los estudiantes en programas y procesos educativos de alta calidad reconocidos nacional e internacionalmente; de esta forma, los egresados son altamente competentes y contribuyen de forma efectiva al desarrollo sustentable de su entorno" (UAA, 2016b: 88).
En este contexto, actualmente la docencia en la UAA se desarrolla en los niveles medio superior, superior y posgrado en 10 centros y 65 departamentos académicos, y está apoyada de manera directa por las áreas administrativas de Docencia de Pregrado, Investigación y Posgrado, Difusión y Vinculación, y Servicios Educativos (UAA, 2016a).

\section{Las raíces: Dirección General de Asuntos Académicos}

Las áreas administrativas antes mencionadas tienen su origen en el Instituto Autónomo de Ciencias y Tecnología (IACT) antes de su transformación en UAA, particularmente en las funciones desarrolladas por los departamentos (IACT, s/f) de: Psicopedagógico, Incorporación y Revalidación de Estudios, Difusión Cultural, Actividades Extracurriculares, Promoción Deportiva y Educación Audiovisual. Lo anterior sirvió como base para proponer en el proyecto de transformación del IACT a UAA, la creación de la Dirección General de Asuntos Académicos, como un área encargada de "diseñar los cursos y carreras, especificar los métodos particulares de 
enseñanza para cada caso y la utilización de los recursos auxiliares [...] [así como] adoptar los métodos de evaluación" (UAA, 2009: 99).

Al formalizarse legalmente el nacimiento de la Universidad Autónoma de Aguascalientes con la publicación de su primera Ley Orgánica y Estatuto (UAA, 1974), las funciones planteadas para la Dirección General de Asuntos Académicos correspondieron al diseño de planes y programas de estudio, selección y diseño de material didáctico, evaluación de la enseñanza, incorporación de instituciones, formación de los profesores, entre otras.

Personal de la Dirección General de Asuntos Académicos, coordinado por el doctor Luis Manuel Macías López.

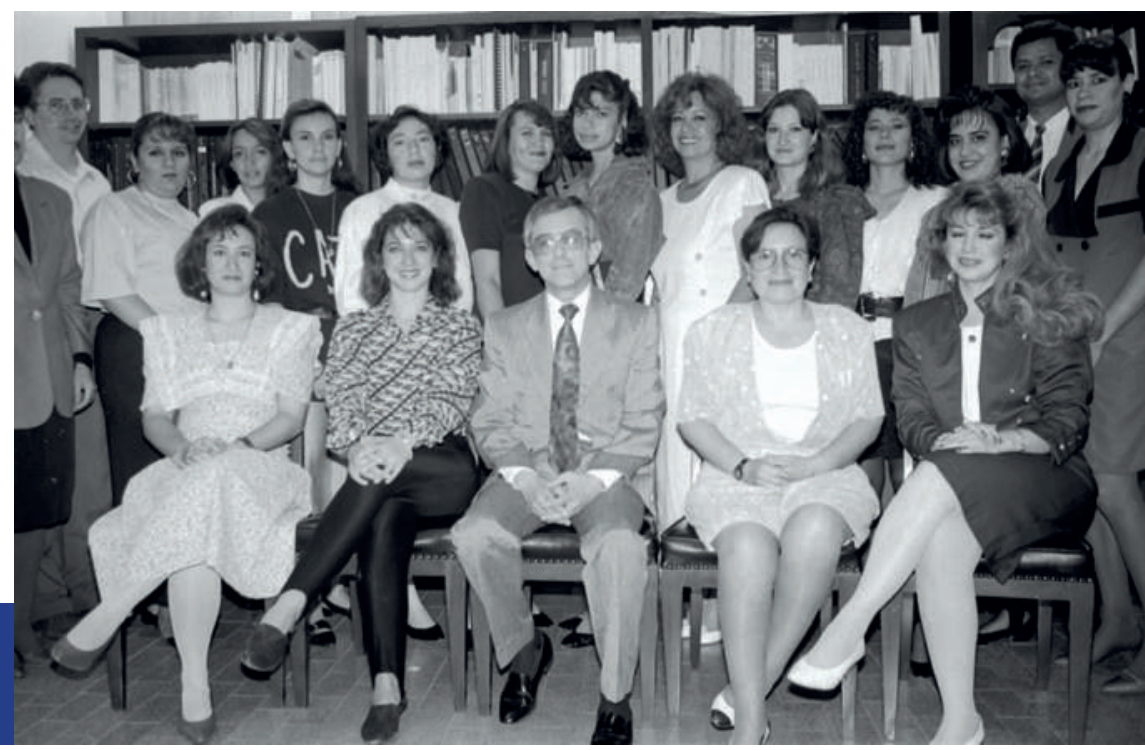

Posteriormente, en 1977 se realizó una reforma a la estructura orgánica de la institución con el propósito de mejorar el funcionamiento del sistema departamental; la Dirección General de Asuntos Académicos fue sustituida por un área denominada "Staff Académico", que "retomaría funciones [de aquella] pero sin el peso que ésa tenía, al comprender a los coordinadores de todas las carreras que ofrecía la Universidad" (Martínez et al., 2013: 170).

No obstante, en 1980:

[...] pudo verse que la UAA sí necesitaba un área para coordinar docencia, investigación y extensión; por ello la reforma del Estatuto estableció de nuevo la Dirección General de Asuntos Académicos, con tres departamentos que, evitando la concentración de recursos, deberían jugar un papel estratégico de impulso y coordinación de esas actividades en todos los centros de la institución (Martínez et al., 2013: 185).

En ese sentido, las funciones principales de esta área de apoyo a los Centros Académicos fueron el diseño y actualización de planes de estudio, la formulación de las políticas de investigación, la planeación de actividades de extensión universitaria, evaluación del personal académico, entre otras (UAA, 1980). En congruencia con estas funciones, la Dirección General de Asuntos Académicos se estructuró en 1980 con los siguientes departamentos: Apoyo a la Docencia, Apoyo a la Extensión y
Apoyo a la Investigación e Intercambio Académico (UAA, 1982). En 1983 se crearon otros dos departamentos: Asesoría Psicopedagógica y Orientación Vocacional, y Videoproducción Docente, además de adecuarse los nombres de dos de los departamentos iniciales para quedar de la siguiente manera: Apoyo a la Docencia e Intercambio Académico, y Apoyo a la Investigación y Educación Continua (UAA, 1985).

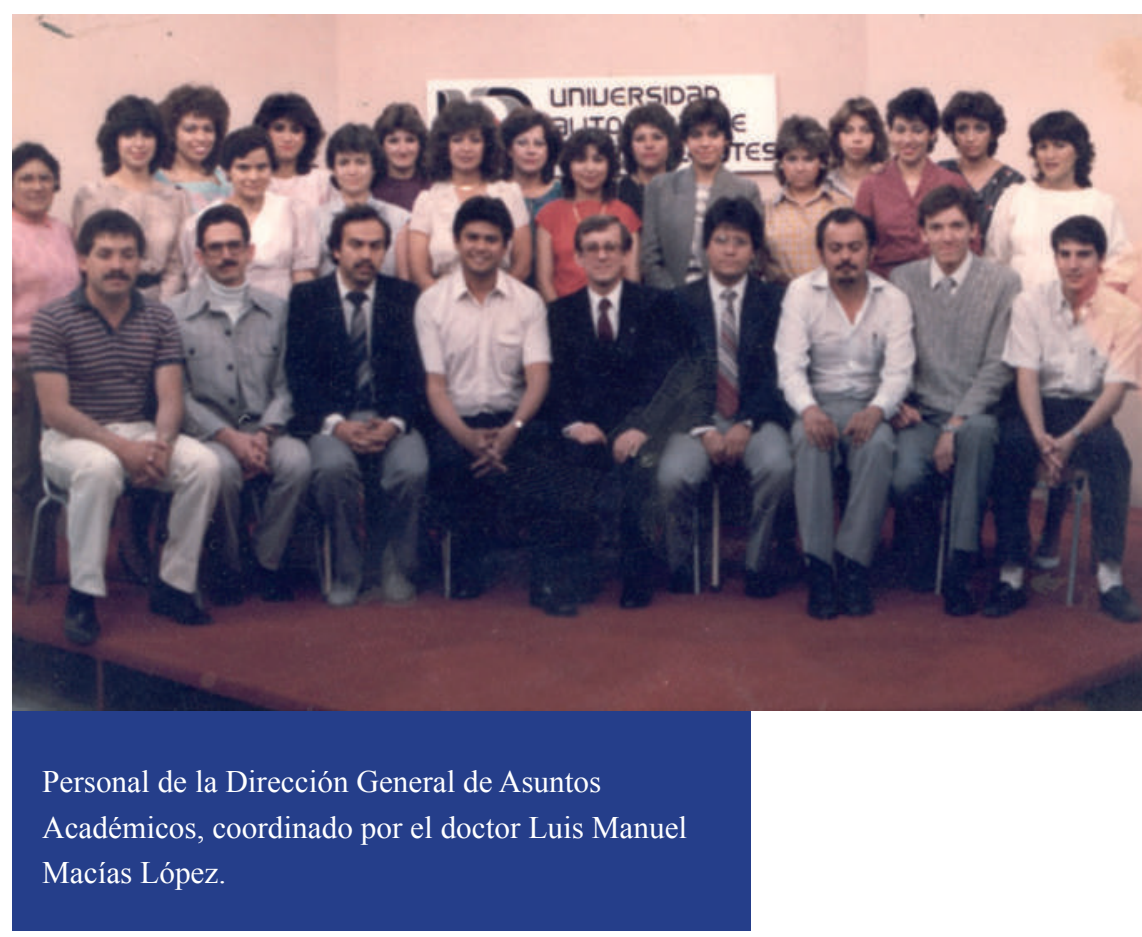




\section{El origen de la Dirección General de Docencia de Pregrado}

Durante el periodo de 1996-1998, se efectuaron un conjunto de reformas normativas a la planeación y a la estructura orgánica de la Universidad Autónoma de Aguascalientes. Producto de ello, la Dirección General de Asuntos Académicos desapareció para crear en su lugar tres nuevas direcciones generales: Docencia de Pregrado, Investigación y Posgrado, y Difusión ${ }^{1}$ (UAA, 1996).

En el caso de la Dirección General de Docencia de Pregrado (DGDP), su función principal fue "diseñar y ejecutar las políticas y programas institucionales de apoyo a la docencia de pregrado y coordinar programas de apoyo a maestros y alumnos" (UAA, 1998b:27); para ello, se crearon los siguientes departamentos: Apoyo a la Formación Integral, Desarrollo Curricular, Estudios Incorporados, ${ }^{2}$ Formación y Evaluación de Profesores, Orientación Educativa y Deportes (UAA, 1998a).

Finalmente, durante el periodo 2010-2011 se realizaron cambios a la estructura orgánica de la DGDP, creándose la Dirección General de Servicios Educativos principalmente para "coordinar las actividades de apoyo y asistencia a la formación integral de los estudiantes, así como aquellos estudios complementarios y actividades académicas que busquen fortalecer la formación educativa de los estudiantes y su competencia académica" (UAA, 2015: 30), por lo cual, la Dirección General de Docencia de Pregrado desarrolla actualmente las siguientes funciones (UAA, 2015: 29):

- Apoyar técnicamente a comités de diseño y revisión de carreras en la formulación de planes de estudios y métodos de enseñanza.

- Evaluar internamente los programas de pregrado, en los términos que establezca el reglamento respectivo; realizar estudios de resultados; dar seguimiento a los egresados y analizar los datos derivados de los procesos selección de alumnos, de cuya preparación técnica se encargará.

1 Más adelante cambió de nombre a Dirección General de Difusión y Vinculación

2 Más adelante cambió de nombre a Departamento de Evaluación de Programas Académicos.

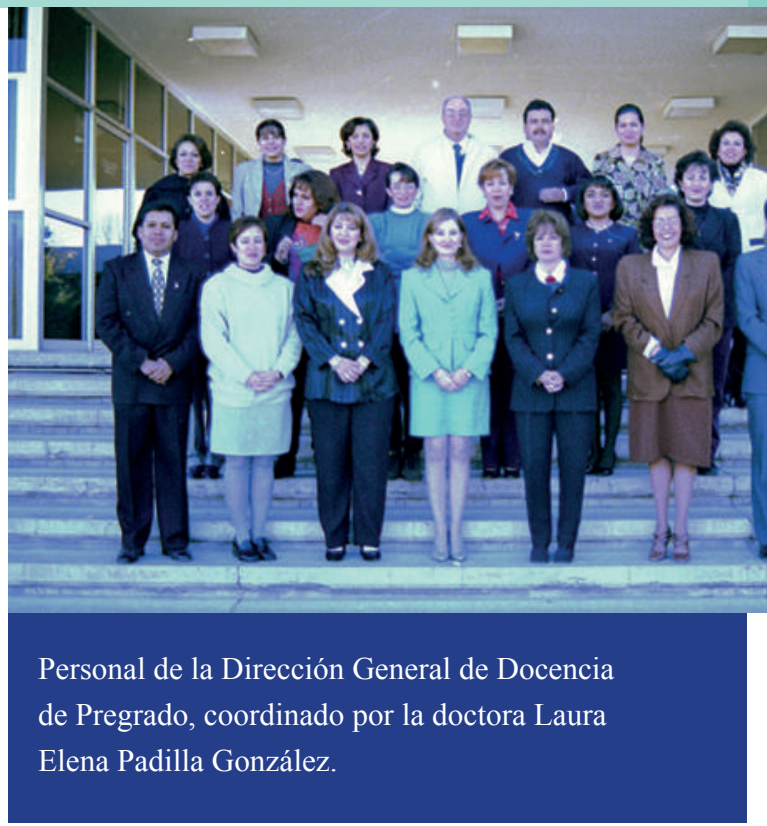

- Coordinar los procesos de evaluación interna y externa de programas de educación media y de pregrado.

- Auxiliar actividades de formación para el personal académico.

- Apoyar a centros y departamentos para la evaluación de los profesores, encargándose de la evaluación semestral por los alumnos de pregrado y posgrado, y de la integración de datos para las evaluaciones bienales.

- Diseñar proyectos de innovación educativa que contribuyan al mejor nivel de logro de los objetivos formativos.

- Coordinar la evaluación de egreso de los estudiantes.

- Evaluar los procesos de enseñanza y de aprendizaje.

- Realizar estudios con empleadores para retroalimentar y actualizar los planes de estudio.

- Coordinar la supervisión de los estudios incorporados.

- Fomentar nuevas modalidades de programas educativos.

Para el desarrollo de estas funciones, la DGDP se estructura actualmente en cinco departamentos: Control y Seguimiento Docente, Desarrollo Curricular, Evaluación Educativa, Formación y Actualización Académica, e Innovación Educativa (UAA, 2016a). 


\section{2}

\section{Conclusión}

Las funciones que actualmente desarrolla la Dirección General de Docencia de Pregrado tienen su raíz y origen en la Dirección General de Asuntos Académicos, misma que fue diseñada desde el proyecto de transformación del Instituto Autónomo de Ciencias y Tecnología a la Universidad Autónoma de Aguascalientes, a partir de las actividades realizadas por algunos departamentos del IACT.

El crecimiento de la UAA en el número de estudiantes, profesores y programas educativos, entre otros factores, derivó en la necesidad de crear nuevas áreas administrativas a partir de las funciones que desempeñaba la Dirección General de Asuntos Académicos, una de ellas fue la Dirección General de Docencia de Pregrado.

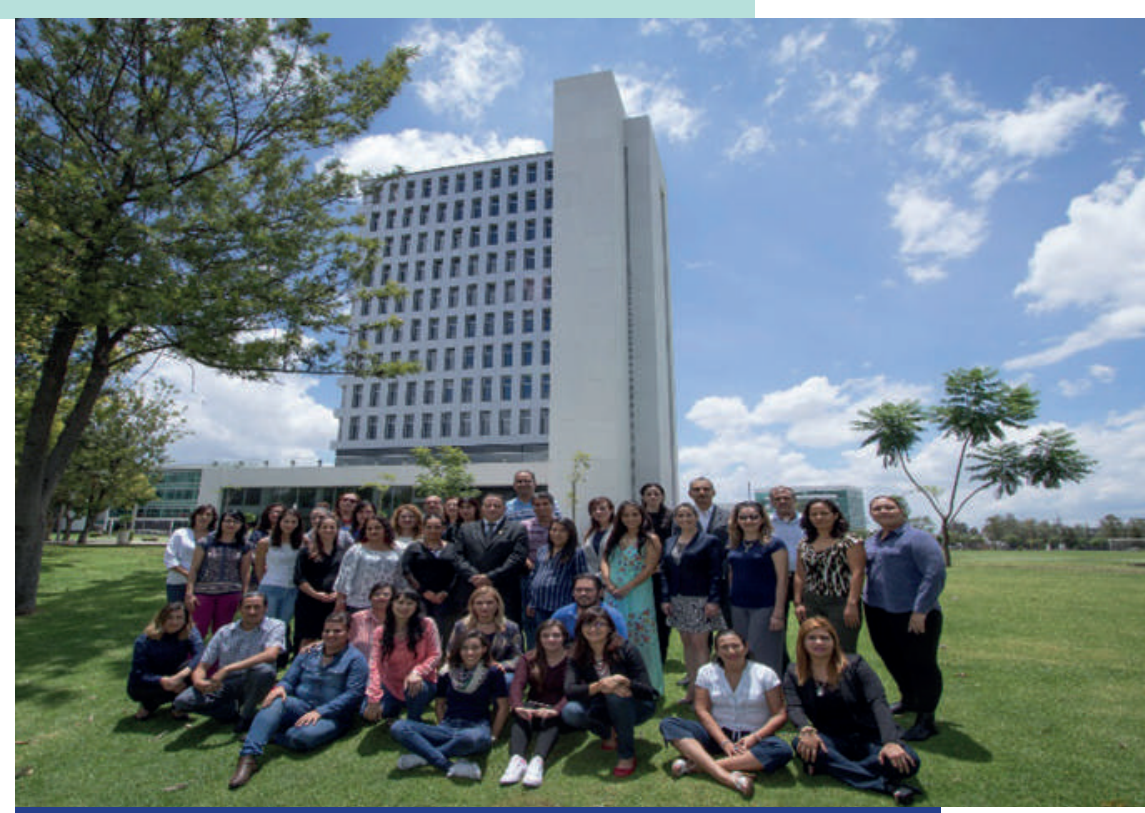

Personal de la Dirección General de Docencia de Pregrado, coordinado por el maestro Juan José Shaadi Rodríguez.

\section{Fuentes de consulta}

Instituto Autónomo de Ciencias y Tecnología (s/f). Memoria de las Actividades del Instituto Autónomo de Ciencias y Tecnología. Ejercicio 1972. México: IACT.

Martínez, H., Pérez, A. y Martínez, F. (2013). Quadragesimo. Crónica de un alumbramiento. México: UAA. Universidad Autónoma de Aguascalientes. (1974). Ley Orgánica y Estatuto de la Universidad Autónoma de Aguascalientes. México: UAA.

Universidad Autónoma de Aguascalientes. (1980). Ley Orgánica y Estatuto. En Correo Universitario, 18 de septiembre de 1980. México: UAA.

Universidad Autónoma de Aguascalientes. (1982). Memoria 1978-1980. México: UAA.

Universidad Autónoma de Aguascalientes. (1985). Carta de Organización aprobada por el H. Consejo

Universitario el 11 de marzo de 1983. En Folleto de Información UAA 1985. México: UAA.

Universidad Autónoma de Aguascalientes. (1996). Propuesta de Reformas a la Estructura Orgánica y Normativa de la Universidad Autónoma de Aguascalientes. México: UAA-DGPD (documento mecanoescrito).

Universidad Autónoma de Aguascalientes. (1998a). Carta de Organización aprobada por el H. Consejo Universitario el 19 de agosto de 1997. En Folleto de Información 1998. México: UAA.

Universidad Autónoma de Aguascalientes. (1998b). Ley Orgánica y Estatuto. En Correo Universitario, cuarta época, No. 30, junio de 1998. México: UAA.

Universidad Autónoma de Aguascalientes. (2009). Proyecto Original de la Universidad Autónoma de Aguascalientes 1973. México: UAA.

Universidad Autónoma de Aguascalientes. (2015). Ley Orgánica de la Universidad Autónoma de Aguascalientes. Estatuto de la Ley Orgánica. Consultada el 9 de marzo de 2018 en: https://docsgc.uaa.mx.

Universidad Autónoma de Aguascalientes. (2016a). Carta de Organización. Consultada el 9 de marzo de 2018 en https://docsgc.uaa.mx.

Universidad Autónoma de Aguascalientes. (2016b). Plan de Desarrollo Institucional 2016-2024. Trazando el Rumbo Institucional. México: UAA. 